Original paper

\title{
Towards MR-guided electron therapy: Measurement and simulation of clinical electron beams in magnetic fields
}

\author{
R. Kueng ${ }^{\mathrm{a}, *}$, B.M. Oborn ${ }^{\mathrm{b}, \mathrm{c}}$, N.F. Roberts ${ }^{\mathrm{b}}$, T. Causer ${ }^{\mathrm{b}, \mathrm{c}}$, M.F.M. Stampanoni ${ }^{\mathrm{d}}$, P. Manser ${ }^{\mathrm{a}}$, \\ P.J. Keall ${ }^{\mathrm{e}}$, M.K. Fix ${ }^{\mathrm{a}}$ \\ ${ }^{a}$ Division of Medical Radiation Physics and Department of Radiation Oncology, Inselspital, Bern University Hospital, and University of Bern, Bern, Switzerland \\ ${ }^{\mathrm{b}}$ Centre for Medical Radiation Physics, University of Wollongong, Wollongong, NSW, Australia \\ ${ }^{\mathrm{c}}$ Illawarra Cancer Care Centre, Wollongong Hospital, Wollongong, NSW, Australia \\ ${ }^{\mathrm{d}}$ Institute for Biomedical Engineering, University of Zurich and Swiss Federal Institute of Technology (ETH), Zurich, Switzerland \\ ${ }^{\mathrm{e}}$ ACRF Image X Institute, Sydney Medical School, University of Sydney, Australia
}

A R T I C L E I N F O

\section{Keywords:}

Electron therapy

Magnetic fields

MRI-linac

Monte Carlo

\begin{abstract}
A B S T R A C T
Purpose: In the current era of MRI-linac radiotherapy, dose optimization with arbitrary dose distributions is a reality. For the first time, we present new and targeted experiments and modeling to aid in evaluating the potential dose improvements offered with an electron beam mode during MRI-linac radiotherapy.

Methods: Small collimated ( $1 \mathrm{~cm}$ diameter and $1.5 \times 1.5 \mathrm{~cm}^{2}$ square) electron beams $(6,12$ and $20 \mathrm{MeV})$ from a clinical linear accelerator (Varian Clinac 2100C) are incident perpendicular and parallel to the strong and localized magnetic fields $(0-0.7 \mathrm{~T})$ generated by a permanent magnet device. Gafchromic EBT3 film is placed inside a slab phantom to measure two-dimensional dose distributions. A benchmarked and comprehensive Monte Carlo model (Geant4) is established to directly compare with experiments.

Results: With perpendicular fields a 5\% narrowing of the beam FWHM and a $10 \mathrm{~mm}$ reduction in the $15 \%$ isodose penetration is seen for the $20 \mathrm{MeV}$ beam. In the inline setup the penumbral width is reduced by up to $20 \%$, and a local central dose enhancement of $100 \%$ is observed. Monte Carlo simulations are in agreement with the measured dose distributions ( $2 \%$ or $2 \mathrm{~mm})$.

Conclusion: A new range of experiments have been performed to offer insight into how an electron beam mode could offer additional choices in MRI-linac radiotherapy. The work extends on historic studies to bring a successful unified experimental and Monte Carlo modeling approach for studying small field electron beam dosimetry inside magnetic fields. The results suggest further work, particularly on the inline magnetic field scenario.
\end{abstract}

\section{Introduction}

The pursuit of clinical success in radiation therapy is a constant trade-off between delivering sufficiently high dose levels to a target region while sparing surrounding healthy tissue. The establishment of image guided radiation therapy (IGRT) in the last two decades has enabled more accurate and reproducible patient setup, which allows treatment planning with smaller margins, yielding improved treatment success [1-3]. As an alternative to the well-established imaging methods with $\mathrm{kV}$ and MV photons, magnetic resonance imaging (MRI) has gained broad interest in radiation therapy over the past years. MR guided radiation therapy (MRgRT) has major advantages in soft tissue contrast compared to $\mathrm{kV}$ and MV imaging, which, combined with high time resolution, has the potential of non-invasive, non-ionizing, on-line image guidance during treatment [4,5]. Additionally to patient setup based on actual position and shape, motion tracking on arbitrary planes during treatment becomes possible. This allows real-time guidance and monitoring of anatomical changes for adaptive dose optimization $[6,7]$. Four main research programs have driven the development of integrated MRgRT systems over the past decade: The Australian MRIlinac program [8], Edmonton's rotating bi-planar system [9], Utrecht's system [10] (now commercialized as the Elekta Unity system) and the ViewRay system [11]. While the ViewRay system was first released as an integrated MRI-Cobalt machine, all four projects have focused their developments towards integrated MRI and linear accelerator designs.

Although these integrated systems are currently intended for photon radiation therapy, the technical prerequisites for treatment with electron beams are fundamentally provided by the linac design. Standard

\footnotetext{
* Corresponding author.

E-mail address: reto.kueng@insel.ch (R. Kueng).
} 
electron treatments in clinical routine are nowadays still performed using standard or patient-specific cut-outs that are mounted into an electron applicator. It has been shown that using the conventional photon multileaf collimator (pMLC) for clinical electron beams at reduced source to surface distances (SSD) can replace treatment plans using cumbersome and inefficient cut-out collimation with similar plan quality for today's standard electron treatments [12]. Alongside advantages in efficiency and quality assurance, using the pMLC for electron beam collimation also allows for advanced treatment techniques like modulated electron radiotherapy (MERT) [13-16], or the combination of photon and electron beams in mixed beam radiotherapy (MBRT) [17-19].

Historically, researchers have also investigated the use of magnetic fields to improve the dosimetry characteristics of electron beams through gross Lorentz force deflections. These studies can be broadly summarized into two areas with slightly different goals: (1) local dose enhancement through local transverse magnetic fields, and (2) lateral dose spread reduction via inline local or globally encompassing magnetic fields.

For local transverse fields there has been a wide variety of both experiments and simulation based studies. By adding a strong and local transverse magnetic field near the end range of a clinical electron beam there is the chance to encourage the electrons to stop in a reduced distance from the surface, thereby giving rise to a local dose enhancement effect. Of the many studies, hypothetical magnetic fields were modeled [20-22], real dipole magnets were used [23-26], or modeling of realistic magnet systems employed [27]. This direction of research has all but ceased to exist in the current era. Put simply, it appears impractical to generate the types of local transverse magnetic fields that would significantly improve current electron beam therapy techniques.

For inline or longitudinal fields, there is only a small collection of studies concerned with modern practical therapeutic electron beams. In 1985 Weinhous simulated several coil magnet devices surrounding a water phantom and with $20 \mathrm{MeV}$ and $30 \mathrm{MeV}$ electron beams of $10 \mathrm{~cm}$ diameter incident [28]. The coil devices had strong fringe gradients that altered the electron beam properties before they reached the phantom, and peaked at around $3 \mathrm{~T}$ inside the phantom. The fields were radically different from pure global inline fields. Hence strong changes to the electrons occurred before the phantom, and the dose changes modeled in the phantom were more of an effect of this process. In 1993 Bielajew simulated $20 \mathrm{MeV}$ pencil beams in 3 and $20 \mathrm{~T}$ uniform global fields [29]. Broad beam doses were emulated by using the dose from a single pencil beams and applying the Geometry-Equivalence Theorem [30] throughout a broad phantom. There was a prediction of a distinct penumbral narrowing for the emulated broad beam. In 2001 Litzenberg performed experiments with a $3.5 \mathrm{~T}$ superconducting solenoid magnet and $10 \mathrm{~cm}$ circular electron beams of $10,20,30,40$, and $50 \mathrm{MeV}$ [31]. The fringe field of the magnet caused significant upstream focusing of the electron beams and so it was not possible to distinguish the impact of the local field in the phantom versus the effects from upstream focusing. Finally in 2005 Chen performed a simulation study of the 2001 Litzenberg work in order to gain insight into the various unexplained results of the experiments. The simulation results confirmed the theory regarding the dose enhancement being associated with external beam focusing. In summary, there is no clear experimental work to confirm the 1993 predictions by Bielajew, nor studies of field strength and arrangements similar to modern day MRI-linac system prototypes. For example around 0.5 to $1.5 \mathrm{~T}$ in strength and of reasonably high uniformity surrounding the phantom.

The purpose of this work is to establish a comprehensive experimental and corresponding in-silico setup to robustly determine dose distributions of small clinical electron beams in the presence of various magnetic field arrangements. The work is targeted to bring new information that is highly relevant for the modern day themed research directions such as optimized dose planning and modulated electron beams, all in the overarching theme of real-time MRI-guidance with a potential electron beam mode.

The novelty is specifically to:

i) Investigate experimentally the changes to small clinical electron beams in magnetic fields with strengths and directions associated with current MRI-linac systems, such that the dosimetry features can be attributed purely to the local magnetic field effects, rather than from beam transport artifacts as seen in previous studies. This is achieved via the use of specially designed magnetic field apparatus that creates a unique local magnetic field that surrounds the dosimetry phantom.

ii) Present a benchmarked and robust magnetic field capable Monte Carlo framework that includes a commissioned electron beam model coupled with finite element magnetic field data of the real experimental device.

\section{Methods}

\subsection{Transverse and inline magnetic field setup}

A permanent magnet device called Magnetic Apparatus for RaDiation Oncology Studies (MARDOS) as introduced by Oborn et al. [32] is used to generate a magnetic field up to $0.713 \mathrm{~T}$ over a volume of several litres, large enough to encompass a small phantom. The two banks of $\mathrm{NdFeB}$ permanent magnets are held in a steel yoke and focusing cones can be inserted to concentrate the magnetic flux across the pole gap. Moving the magnet banks towards and apart from each other as well as inserting the cones changes the magnetic field distribution and maximum field strength of the setup.

The MARDOS system was designed by optimizing COMSOL Multiphysics finite element models of two banks of NdFeB permanent magnets incorporated in a steel frame or yoke. Once constructed, the magnetic performance of the system was mapped out and the data used to fine tune the magnet and steel grade values in the model, such that it matches the experimental performance. The fields were measured with a MAGSYS Gaussmeter HGM09s which has a calibration accuracy of $\pm 0.5 \%$ over the range of $0-1 \mathrm{~T}$. The end result was that there is a $2 \%$ match to the magnetic field values between the banks over all combinations of bank settings. During any experimental work, the banks are adjusted to predetermined positions and the magnetic field strength between the cones spot checked with a portable field probe to ensure the setup matches the benchmarked modeling scenarios.

MARDOS can be rotated in order to align the primary electron beam direction perpendicular (referred to as transverse setup) or parallel (referred to as the inline setup) to the main magnetic field direction. Solid water slabs with a size of $30 \times 30 \mathrm{~cm}^{2}$ and thickness of 0.1 to $5 \mathrm{~cm}$ are stacked in the MARDOS system and irradiated parallel to the slab interfaces (transverse setup) and perpendicular to the slab interfaces (inline setup). Figs. 1 and 2 show the different applied setups of the MARDOS system. For all measurements, the MARDOS system is positioned such that a source to magnet isocenter distance (SID) of $150 \mathrm{~cm}$ is achieved. Electron beams $(6,12$ and $20 \mathrm{MeV})$ are produced using a Varian Clinac 2100C linear accelerator and 2000 monitor units are applied for each measurement. Gantry position is at 270 degrees for all MARDOS measurements and the electron beam is collimated with the photon MLC to a $10 \times 10 \mathrm{~cm}^{2}$ field size defined at machine isocenter (at $100 \mathrm{~cm}$ ). Final collimation of the electron beam before entering the magnetic field of the MARDOS system is achieved by different physical components for the different setups. Without the focusing cones in place, final collimation is performed with a drilled cerrobend mold, yielding a circular collimation of $1 \mathrm{~cm}$ in diameter. For the setup with focusing cones in place, final collimation is achieved with a square window of $1.5 \times 1.5 \mathrm{~cm}^{2}$ at the cone tips.

The fringe fields were also modeled and measured outside of the steel yoke and found to be less than $0.001 \mathrm{~T}$. This has negligible impact on electron beam transport, for example the radius of curvature for a 

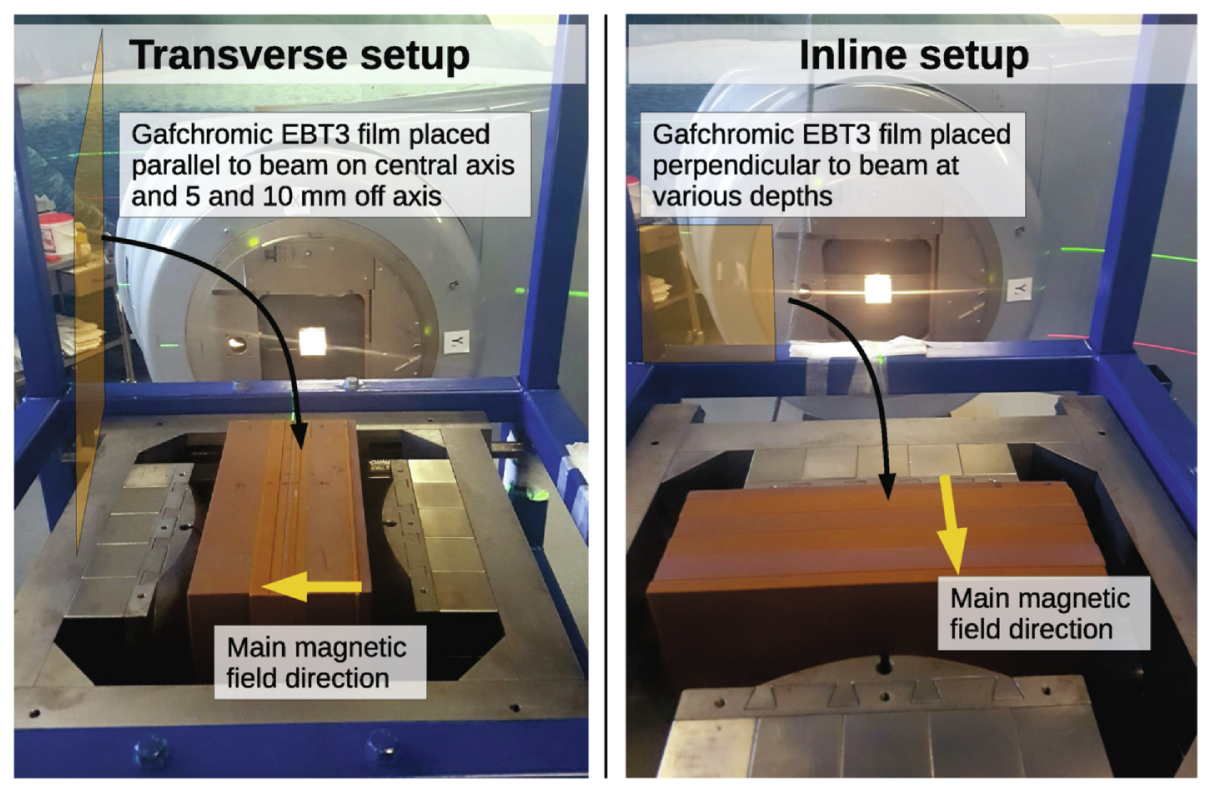

Fig. 1. Placement of the solid water slabs within the MARDOS system for the transverse (left) and inline without cones (right) setup. Gafchromic EBT3 film pieces are placed parallel (left) and perpendicular (right) to the primary beam direction at different off-axis distances and depths, respectively.

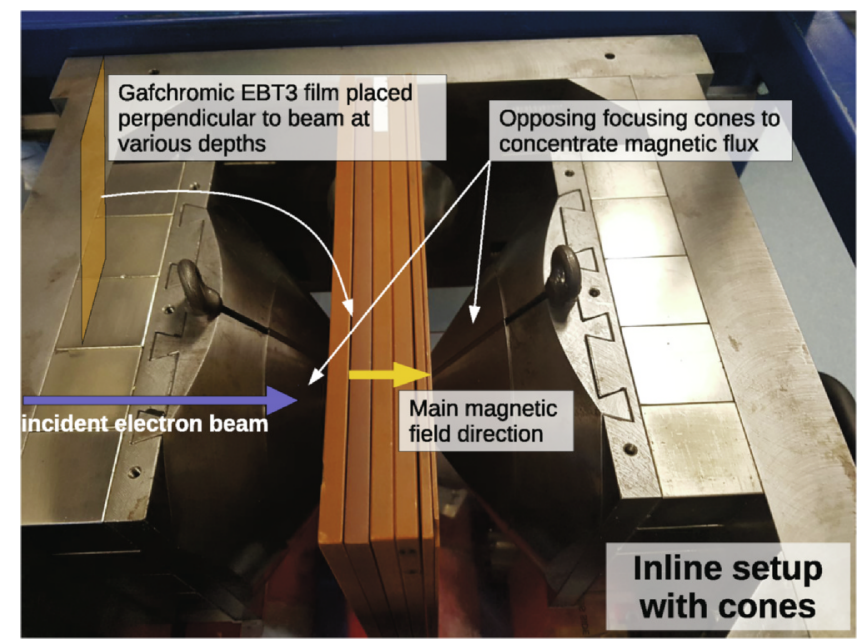

Fig. 2. Placement of the solid water slabs within the MARDOS system for the inline setup with cones. Gafchromic EBT3 film pieces are placed perpendicular to the primary beam direction at different depths.

$6 \mathrm{MeV}$ electron traveling in a $0.001 \mathrm{~T}$ magnetic field is $21.6 \mathrm{~m}$.

\subsection{Zero magnetic field reference setup}

To establish reference conditions with no magnetic field, all measurements performed within the magnet are repeated without the MARDOS magnet. These $0 \mathrm{~T}$ reference experiments are performed by mounting the collimation hardware and phantom inside a dedicated magnetic field free aluminum replica of the MARDOS frame. To reproduce the setup with focusing cones, the cones are removed from the MARDOS device and mounted in the replica frame.

\subsection{Film measurements}

Gafchromic EBT3 film (Ashland, Convington, USA) placed in the solid water slab phantom is used for dose measurements in transverse, inline and zero magnetic field. The film is calibrated for a dose range of 0-10 Gy following the protocol outlined by Devic et al. [33] using a standard single-channel analysis procedure (red channel) to convert net optical density to dose. The film are scanned on an Epson Expression 10000XL flatbed scanner with 72 dpi resolution. Pieces of film with dimensions of $10 \times 25 \mathrm{~cm}^{2}$ and $5 \times 5 \mathrm{~cm}^{2}$ are irradiated in the transverse and inline setup, respectively, in the MARDOS magnet as well as in the zero magnetic field reference setup. Fig. 3 shows an illustration of the film placement in the transverse setup with indication of the incident electron beam and expected beam deflection due to Lorentz force.

For the inline setup with and without cones, the $5 \times 5 \mathrm{~cm}^{2}$ pieces of film are positioned such that the impinging electron beam is aligned with the center of the film. Table 1 shows an overview of the acquired film measurements in the different setups.

No measurements are performed in the transverse setup with a maximum magnetic flux density of $0.713 \mathrm{~T}$, as the electron beam would not reach the phantom due to the deflection caused by the Lorentz force.

\subsection{Monte Carlo framework}

For characterization of the electron beams, a multiple source MC beam model as described by Henzen et al. [16] is commissioned and validated for the Varian Clinac 2100C for the three applied electron energies $(6,12$ and $20 \mathrm{MeV})$. The beam model consists of a foil and a jaw source, both divided into an electron and a photon part. For the beam model commissioning of a specific treatment unit, depth dose and air profile measurements at an SSD of $70 \mathrm{~cm}$ are collected and

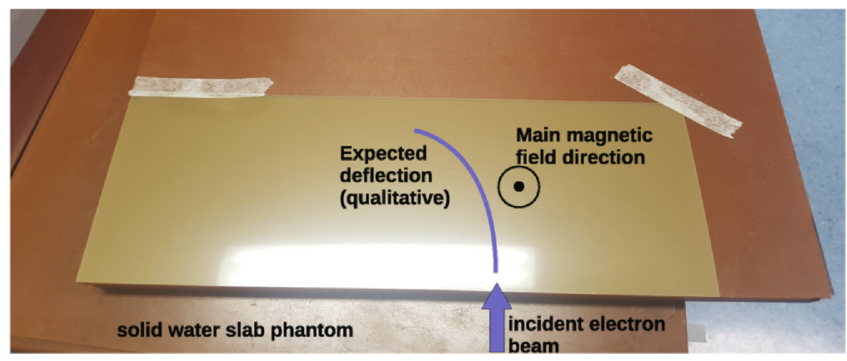

Fig. 3. Gafchromic EBT3 film as irradiated in the transverse magnetic field setup. Pieces of film are placed on the central axis plane as well as $5 \mathrm{~mm}$ and $10 \mathrm{~mm}$ off-axis for all measurements in the transverse setup. 
Table 1

Overview of the acquired film measurement data in the magnetic field setup.

\begin{tabular}{|c|c|c|c|c|}
\hline Energies & Collimation & Magnet setup & $B_{\max }$ & Film measurements \\
\hline $6,12,20 \mathrm{MeV}$ & $1 \mathrm{~cm}$ circular & transverse & $0.265 \mathrm{~T}$ & Central axis plane, 5 and $10 \mathrm{~mm}$ off-axis \\
\hline $6,12,20 \mathrm{MeV}$ & $1 \mathrm{~cm}$ circular & transverse & $0.353 \mathrm{~T}$ & Central axis plane, 5 and $10 \mathrm{~mm}$ off-axis \\
\hline $12 \mathrm{MeV}$ & $1 \mathrm{~cm}$ circular & inline & $0.353 \mathrm{~T}$ & Perpendicular @1, 2, 3, 4, 5 cm depth \\
\hline $20 \mathrm{MeV}$ & $1 \mathrm{~cm}$ circular & inline & $0.353 \mathrm{~T}$ & Perpendicular@ @1, 3, 5, 7, 9 cm depth \\
\hline $6 \mathrm{MeV}$ & $1.5 \mathrm{~cm}$ square & inline with cones & $0.713 \mathrm{~T}$ & Perpendicular @1,2 cm depth \\
\hline $12 \mathrm{MeV}$ & $1.5 \mathrm{~cm}$ square & inline with cones & $0.713 \mathrm{~T}$ & Perpendicular@1, 2, 3, 4 cm depth \\
\hline
\end{tabular}

combined with pre-calculated MC configuration data. The beam model is connected to the Swiss Monte Carlo plan (SMCP) [34], where particle transport through the pMLC is performed with the PIN MC algorithm [35] and phase space files are collected for further particle transport and dose calculation. Magnetic flux maps for the different setups of the magnet system are calculated using finite element calculation using the COMSOL Multiphysics ${ }^{\circledast}$ software (COMSOL AB, Stockholm, Sweden) and are exported as three dimensional lookup tables. The geometric setup of the MARDOS system is modeled and particle transport is simulated using the Geant4 simulation toolkit [36] version 10.2 with standard electromagnetic physics models. The recorded phase space files from the beam model as well as the lookup tables for the magnetic field are loaded in the Geant4 simulation to perform accurate particle transport with and without the magnetic field. Dose is scored at $1 \times$ $1 \times 1 \mathrm{~mm}^{3}$ resolution throughout the phantom volume. An energyspecific normalization factor is determined to calculate absolute dose in units of Gray. The calculated dose distributions are compared to the measurements by means of dose profiles and two-dimensional gamma evaluation [37].

To further assess the impact of an inline magnetic field on an clinical electron beam, an additional MC study is performed. Electron beams of the three commissioned energies 6,12 and $20 \mathrm{MeV}$ are collimated with the pMLC to a circular field with a $10 \times 10 \mathrm{~cm}^{2}$ equivalent field size. A water phantom with unilateral lung and bone inhomogeneities is placed at an SSD of $100 \mathrm{~cm}$ and a homogeneous inline magnetic field of $0.713 \mathrm{~T}$ is activated from $10 \mathrm{~cm}$ upstream of the phantom. The resulting dose distributions are compared to the reference situation without magnetic field in terms of dose enhancement and change of penumbral fall-off.

\section{Results}

\subsection{Magnetic field distribution}

The MARDOS system produces a strong magnetic field encompassing a substantial volume, as displayed in Fig. 4. However importantly for this study, the field distribution has some unique features. In the top figure a homogeneous magnetic flux density with a maximum of $0.265 \mathrm{~T}$ and $0.353 \mathrm{~T}$ is reached with an open gap of $15 \mathrm{~cm}$ and $10 \mathrm{~cm}$, respectively. The iron yoke of the MARDOS system strongly contains the magnetic flux, allowing for electron beams to travel very close to the phantom before entering these strong fields. For the transverse experiments, the electron beam undergoes almost no deflection before striking the phantom. This is evident from the results of the next section, and importantly allows us to examine the local effects of the magnetic field essentially within the phantom volume.

With the steel cones in place (bottom figure), a maximal magnetic flux density of $0.713 \mathrm{~T}$ is obtained over the gap of $5 \mathrm{~cm}$ between the cone tips. In this arrangement for inline experiments, it can be seen that the electron beam experiences almost no magnetic field during its path to the phantom volume. This is key requirement to ensure the effects on the electron beam are induced by inline magnetic field within the phantom rather than any upstream focusing effects.

\subsection{Transverse field results}

Fig. 5 shows measured dose distributions on the central axis plane, the $5 \mathrm{~mm}$ off-axis plane and the $10 \mathrm{~mm}$ off-axis plane for three electron energies in the transverse magnetic field setup for the three electron beam energies. The film data shows a substantial deflection of the electron beam for the three initial energies $(6,12$ and $20 \mathrm{MeV})$ for the two non-zero field strengths $\left(B_{\max }=0.265 \mathrm{~T}\right.$ and $0.353 \mathrm{~T}$, respectively) when compared to the reference measurements without a magnetic field. Also apparent in the $6 \mathrm{MeV}, 0 \mathrm{~T}$ image is an outline of the background photon contamination, or scattered photons produced in the head of the linac. This takes the shape of the final $1 \mathrm{~cm}$ diameter cerrobend collimator outline and it contributes less than $1 \%$ of the primary electron beam dose. It is not evident when the magnetic field is introduced simply due to colormap thresholding changing to account for a small relative dose increase when the magnetic fields are applied. Fig. 6 shows the corresponding isodose curves on the central axis plane. The beam deflection can be observed in all isodose lines and a lateral shift of up to $7 \mathrm{~mm}$ is measured for the lowest indicated isodose line, that is $0.5,1.0$ and $1.5 \mathrm{~Gy}$ for the 6,12 and $20 \mathrm{MeV}$ beam, respectively. This is confirmed by a lateral dose profile in a depth of $1.5 \mathrm{~cm}$, as shown in the bottom right of Fig. 6, where the respective maximum dose value of the profile is shifted by up to $5 \mathrm{~mm}$. For all three electron energies, a drop in the dose is apparent in the isodose lines after initial build-up at a phantom depth of around $0.5 \mathrm{~cm}$. The cause of these artefacts are small air gaps between the slabs of the solid water phantom and the irradiated film, which perturb electron scattering conditions if the film measurement plane is parallel to the incident beam direction [38].

The most important clinical result of the perpendicular work would be considered the changes to the $20 \mathrm{MeV}$. As indicated in Fig. 6, there is a clinically significant reduction of $10 \mathrm{~mm}$ in penetration depth of this beam, as well as a mild reduction of the full width at half maximum (FWHM) at $1.5 \mathrm{~cm}$ depth.

Monte Carlo results are in good agreement with the acquired film measurement, as exemplarily indicated in Fig. 7 for the $12 \mathrm{MeV}$ beam in a $0.353 \mathrm{~T}$ magnetic field. The acquired dose distributions on the central axis plane are shown for the film data (left) and the MC simulation (center) and a gamma map is shown on the right. A global dose difference criterion of $2 \%$ of the maximum measured reference dose, a distance-to-agreement criterion of $2 \mathrm{~mm}$ and a dose threshold of $20 \%$ is applied. Table 2 shows the results of the gamma evaluation on the central axis plane for the measured and simulated dose distributions in the transverse setup.

\subsection{Inline field results: $12 \mathrm{MeV}$}

In the inline setup, for a magnetic field with $B_{\max }=0.353 \mathrm{~T}$ as well as $0.713 \mathrm{~T}$, a dose enhancement of the dose profiles can be observed compared to the corresponding $B=0 \mathrm{~T}$ measurement. Two-dimensional dose distributions of the measured films are shown in Fig. 8 for a $12 \mathrm{MeV}$ electron beam in a $0.713 \mathrm{~T}$ inline magnetic field. For all investigated depths, an increase of the dose can be observed in the presence of a magnetic field compared to the reference condition. This observation is quantitatively shown in the corresponding lateral dose profiles in Fig. 9. An increase of the dose level is prominent for all 

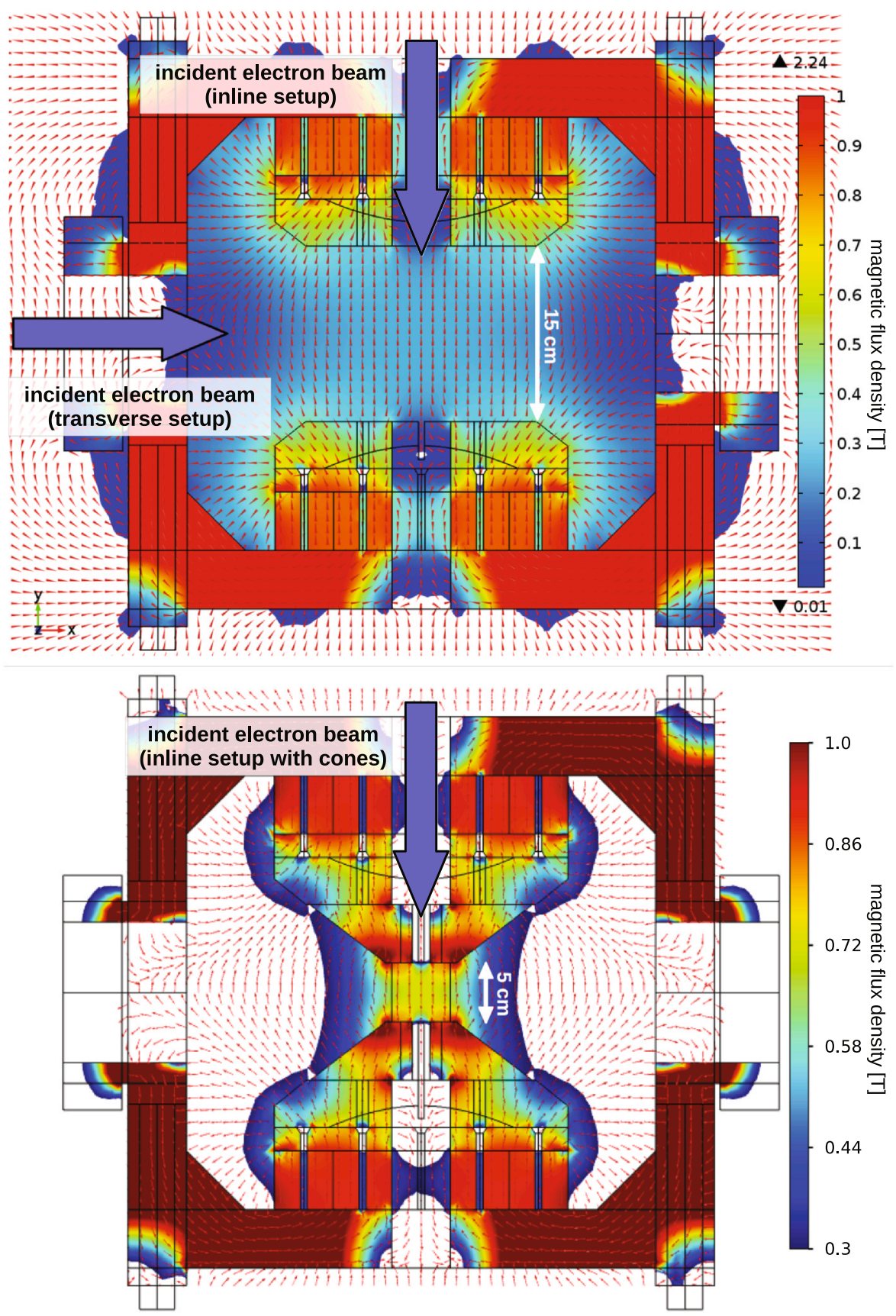

Fig. 4. Top: Top view of the magnetic flux density (color) and direction (red arrows) of the MARDOS system in the setup, where a maximum field strength of $0.265 \mathrm{~T}$ is achieved in the phantom volume. Bottom: Similar view for the inline measurements where the $0.713 \mathrm{~T}$ field is produced over a $5 \mathrm{~cm}$ cubic volume between the cone tips. (For interpretation of the references to color in this figure legend, the reader is referred to the web version of this article.)

measured depths, with a relative dose difference of up to $25 \%$ in $4 \mathrm{~cm}$ depth.

\subsection{Inline field results: $6 \mathrm{MeV}$}

The same effect is present for the $6 \mathrm{MeV}$ beam in the inline setup with cones, where the maximal magnetic field strength is $0.713 \mathrm{~T}$. For the measurements in both $1 \mathrm{~cm}$ and $2 \mathrm{~cm}$ depth, a substantial increase of the dose values can be observed, as indicated by the lateral dose profiles shown in Fig. 10. An increase of measured dose of $100 \%$ is observed with respect to the reference measurement without a magnetic field. This results in a narrowed field size when re-normalizing to a given dose level at the center of the field, for example a decreased
FWHM of $-1.5 \mathrm{~mm}$ in $2 \mathrm{~cm}$ depth compared to $0 \mathrm{~T}$. Additionally, the penumbral fall-off from the $80 \%$ to $20 \%$ dose level is reduced by $-2 \mathrm{~mm}(-20 \%)$. As shown in Figs. 9 and 10, MC results are within $2 \%$ dose differences or $2 \mathrm{~mm}$ distance-to-agreement.

\subsection{Inhomogeneity simulation study results}

Fig. 11 shows the results of the additional MC study of a clinically relevant field size on a phantom including inhomogeneities.

The dose distributions are normalized to the respective dose maximum of the $B=0 \mathrm{~T}$ situation for each energy. A dose increase from and reduction of the penumbral fall-off can be observed for all energies, being most prominent for the $6 \mathrm{MeV}$ electron beam (50\% dose increase 
$6 \mathrm{MeV}$
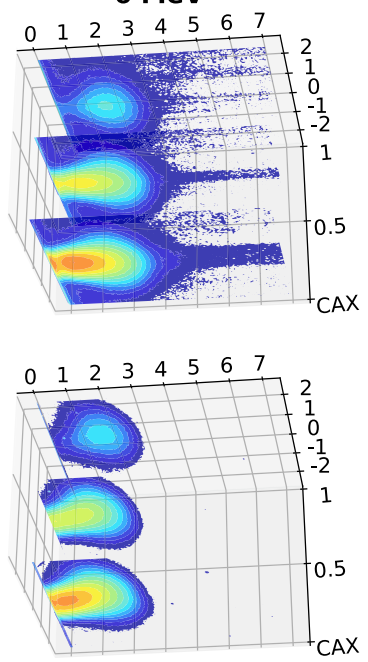

$0.353 \mathrm{~T}$

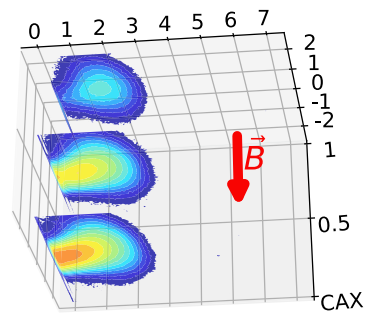

$12 \mathrm{MeV}$
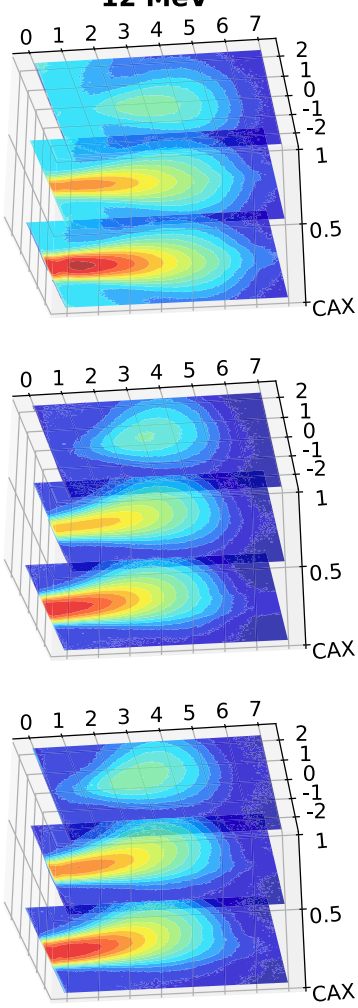

$20 \mathrm{MeV}$
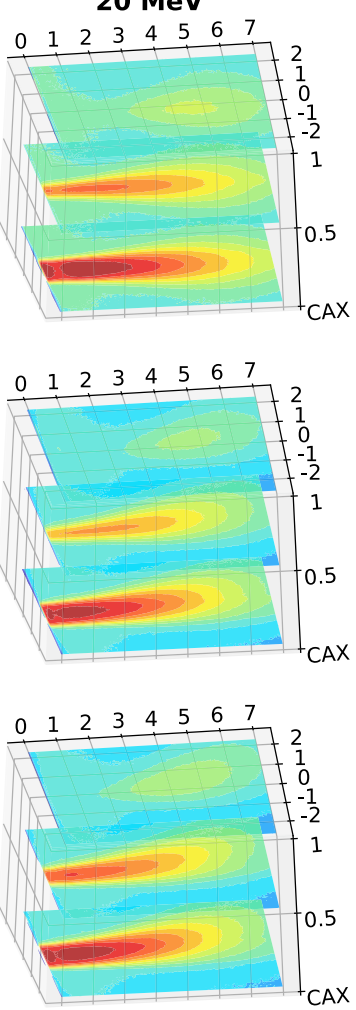

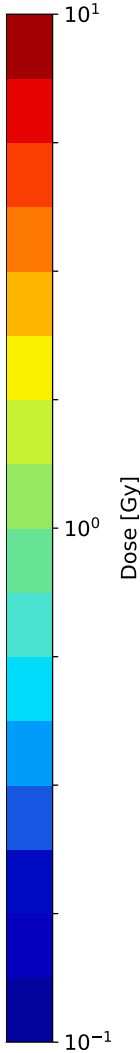

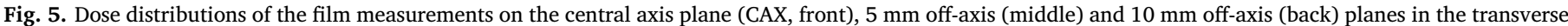

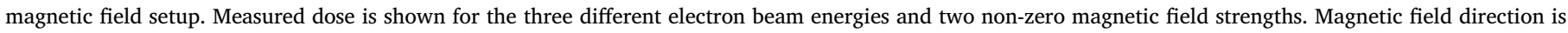
marked in the lower left subplot, axis units are in centimeters.

and $-32 \%$ reduction of penumbral fall-off from $80 \%$ to $20 \%$ in $2 \mathrm{~cm}$ depth) and less pronounced for the higher energies. The results of this in-silico investigation support the potential dosimetric offerings of electron beams in an inline MRI-linac system.
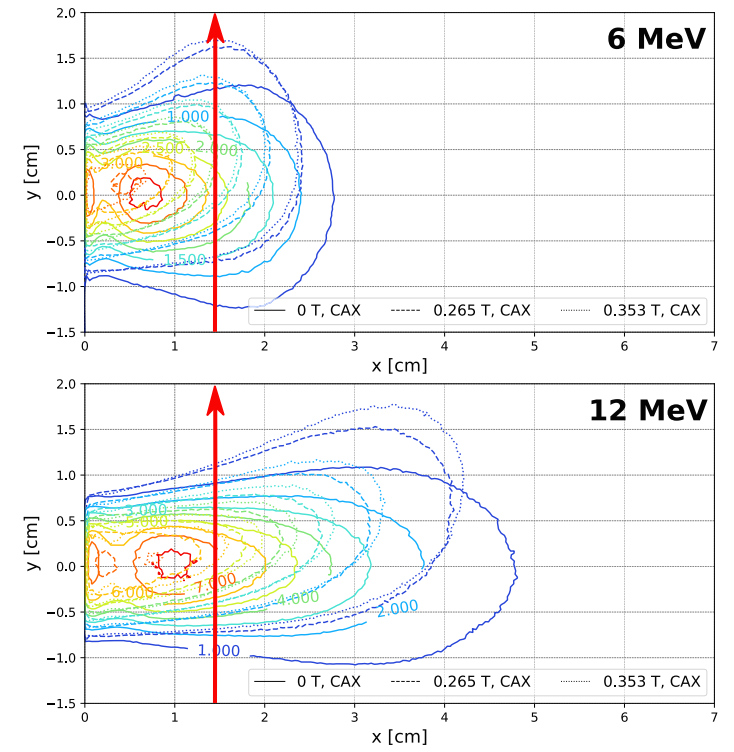

\section{Discussion}

\subsection{Novelty of work}

Despite the wide range of historic research and experiments conducted on radiotherapy electron beams and changes induced by magnetic fields, some simplified scenarios have been overlooked or not
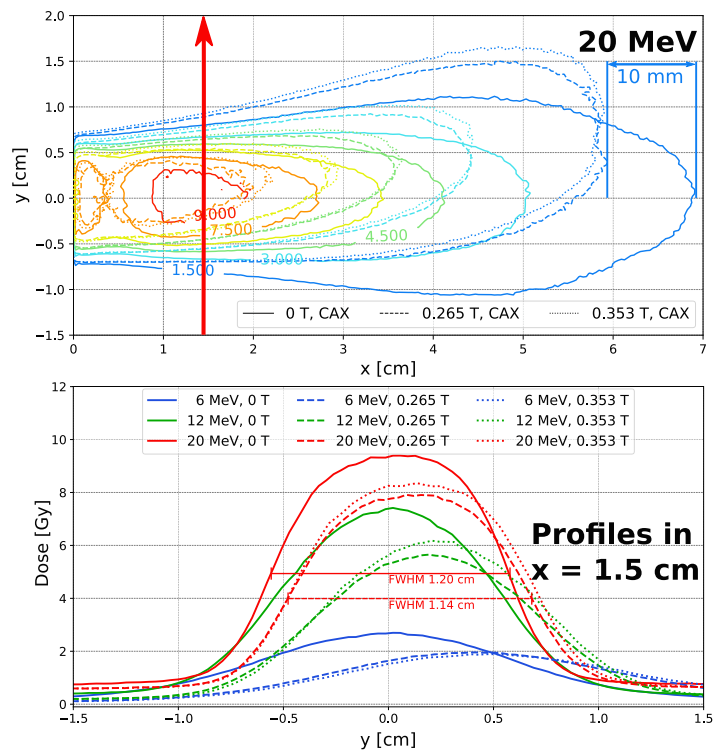

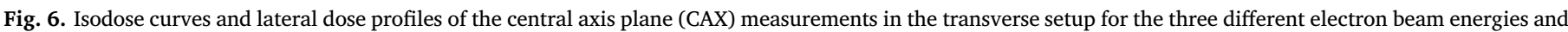

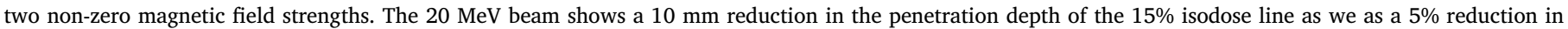
FWHM at $1.5 \mathrm{~cm}$ depth. 

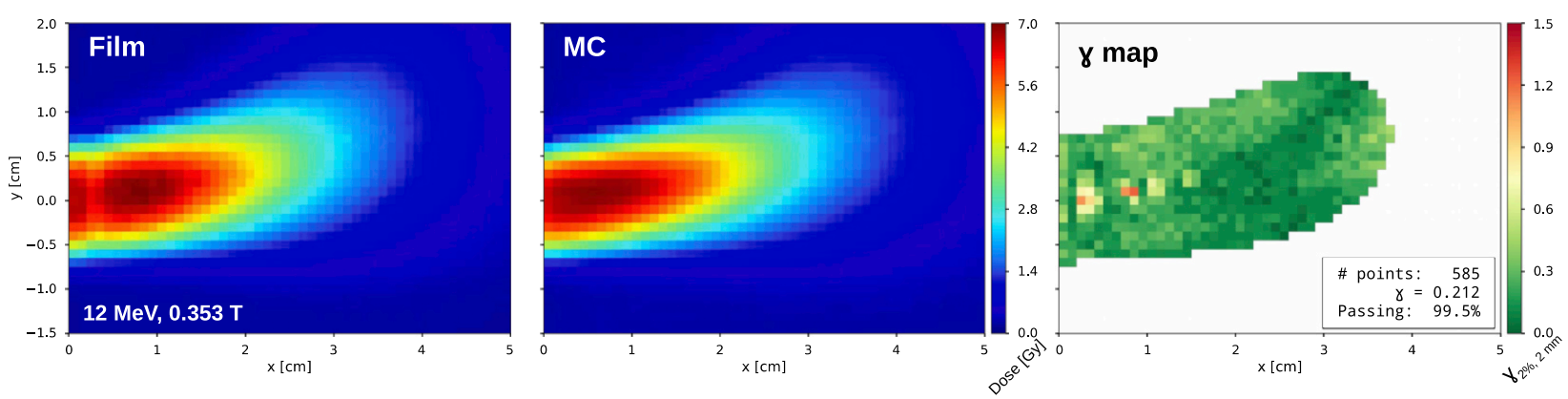

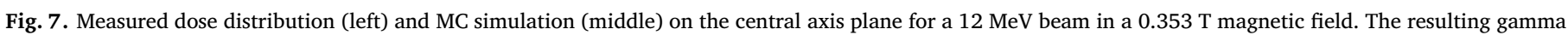
map is shown (right) with a global $2 \%$ and $2 \mathrm{~mm}$ criterion and a dose threshold of $20 \%$.

Table 2

Results of 2D gamma evaluation for the transverse setup. A global $2 \%$ and $2 \mathrm{~mm}$ criterion and a dose threshold of $20 \%$ is applied.

\begin{tabular}{rrrrr}
\hline Energy & $B_{\max }$ & \# points & Mean $\gamma$ & Passing rate \\
\hline $6 \mathrm{MeV}$ & $0 \mathrm{~T}$ & 411 & 0.353 & $93.4 \%$ \\
$6 \mathrm{MeV}$ & $0.265 \mathrm{~T}$ & 386 & 0.451 & $97.2 \%$ \\
$6 \mathrm{MeV}$ & $0.353 \mathrm{~T}$ & 367 & 0.180 & $100.0 \%$ \\
$12 \mathrm{MeV}$ & $0 \mathrm{~T}$ & 594 & 0.242 & $96.0 \%$ \\
$12 \mathrm{MeV}$ & $0.265 \mathrm{~T}$ & 550 & 0.166 & $99.3 \%$ \\
$12 \mathrm{MeV}$ & $0.353 \mathrm{~T}$ & 585 & 0.212 & $99.5 \%$ \\
$20 \mathrm{MeV}$ & $0 \mathrm{~T}$ & 885 & 0.255 & $96.4 \%$ \\
$20 \mathrm{MeV}$ & $0.265 \mathrm{~T}$ & 922 & 0.225 & $99.7 \%$ \\
$20 \mathrm{MeV}$ & $0.353 \mathrm{~T}$ & 914 & 0.327 & $96.9 \%$ \\
\hline
\end{tabular}

verified. For the first time, our experiments of linac-generated electron beams show the minor reductions in electron beam widths caused by inline magnetic fields of $0.713 \mathrm{~T}$. The effect is caused by the local magnetic field rather than by changes to electron paths above the phantom as was the case in the previous studies [39,31].

In particular, our unique experimental apparatus with a very low fringe field has given us the ability to remove any effects of electron beam transport above the phantom.

Our work also brings together a comprehensive in-silico framework using a designated MC electron beam model, finite element calculations to characterize the magnetic field map, and a general purpose MC code to simulate electron beam transport and energy deposition. Importantly the modeling predictions shows results within clinical agreement of the measurements correspondingly performed.

\subsection{Film artefacts}

While the transverse setup shows the expected deflection of the clinical electron beam, artefacts in the dose distributions are observed

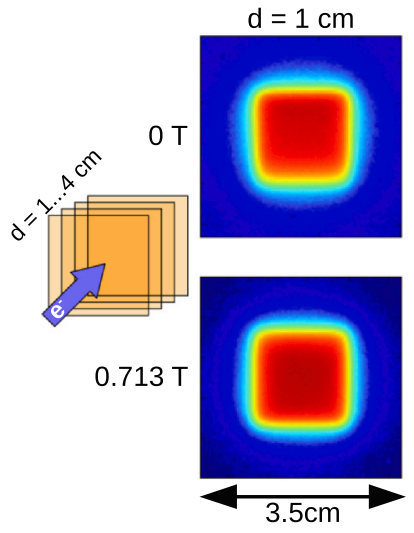

in the superficial region, which is associated with small air gaps. This effect was already described by Dutreix et al. [38], when a film is placed parallel to the primary beam. A designated phantom could be used to minimize such artefacts [40] or a tilting angle of the phantom could be introduced to avoid the primary beam to be exactly parallel to the film plane [41]. Due to the mechanical properties and placement of final collimation in the present experimental setup, such a tilting is not feasible for the measurements in the transverse orientation. However, the observed artefacts do not substantially influence the results presented in this work as confirmed by the MC simulation data.

\subsection{Dose enhancement effects of transverse fields}

Previous work has suggested the use of such strong transverse magnetic fields to ameliorate electron dose distributions [23-26]. To achieve this effect in the context of MRI-linac radiotherapy, the electron beams would have to be shielded from the magnetic field up to the surface to prevent them from spiraling away before reaching the patient. Our results for the transverse magnetic field work has shown indeed how small electron beam dosimetry can be slightly improved through a reduction in low dose penetration and minor width reductions, at the cost of asymmetric beam shape. The MARDOS device allows for an electron beam to travel essentially unperturbed by a magnetic field above the phantom region. Hence we were able to observe the raw effects of a very local transverse magnetic field. In the most realistic sense, it would seem this magnetic field property would not be permittable inside an MRI-linac system. By nature, a patient inside an MRI is required to be surrounded by a highly uniform magnetic field. In this case even a $20 \mathrm{MeV}$ electron could be completely deflected by the MRI field and so not reach the patient. The data presented in this section of work therefore has the most impact for testing the predictions of our multifaceted modeling workflow rather than describing a feasible dose enhancement method using transverse magnetic fields. Our results

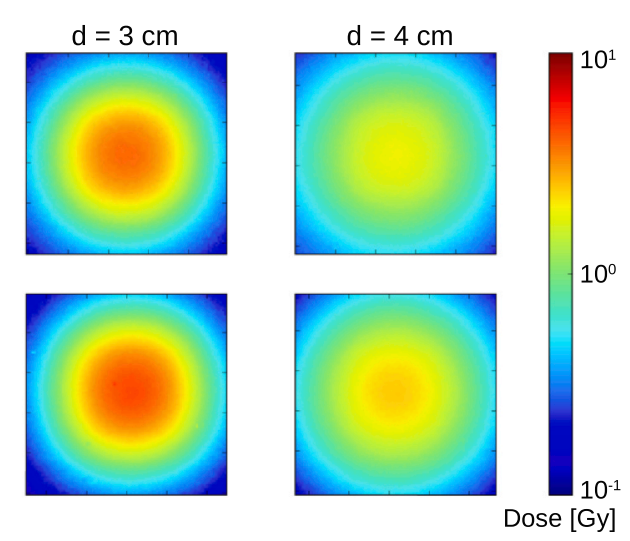

Fig. 8. Dose distributions of the film data placed at depths $(d)$ in the phantom in an inline magnetic field setup for the $12 \mathrm{MeV}$ electron beam. 


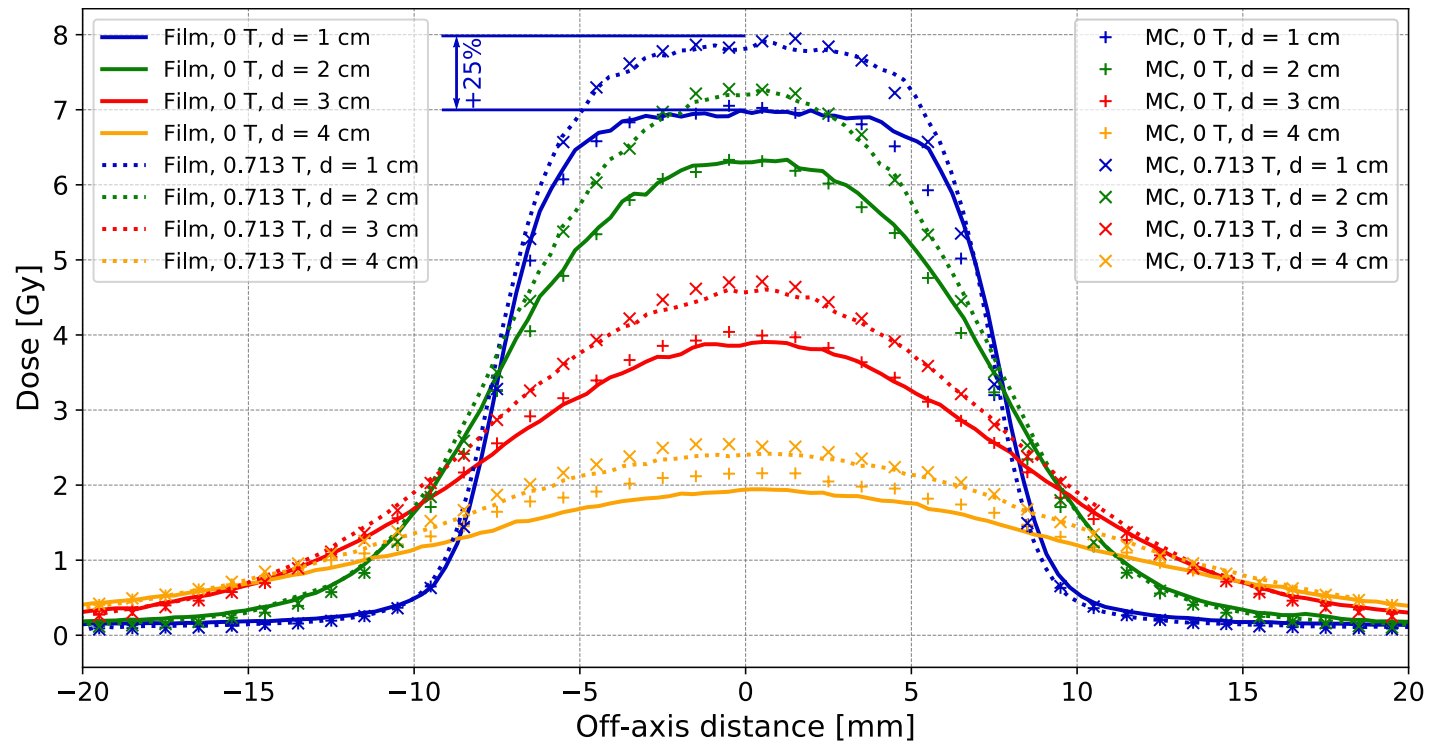

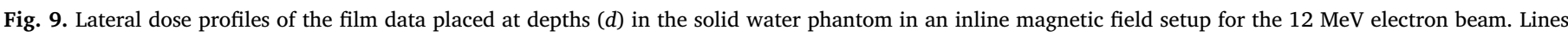

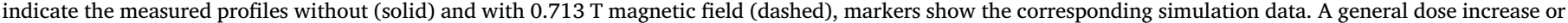
enhancement in the order of $25 \%$ is observed at each depth.

indeed show a good quality match over the various electron beam energies and magnetic field strengths.

\subsection{Dose enhancement effects of inline fields}

On the other hand, the presented results show the enhancement of dose in the inline setup, where the main magnetic field is parallel to the incident electron beam, compared to a reference situation without an external magnetic field. The magnitude of the dose enhancement can be associated in part with the small measured field size, which emphasizes this effect, but the same qualitative characteristics are observed for a clinically more relevant field size. In this inline setup, electrons are not affected by the magnetic field as long as their direction of motion is aligned with the magnetic field lines. As soon as a perpendicular component arises due to beam divergence or scattering, the electron is forced on a helical path around the axis of the magnetic field direction as determined by the Lorentz force. The inline field therefore encourages laterally scattering electrons in the phantom to propagate parallel to the field direction. This results in a narrowed field size of the electron beam and a steeper lateral penumbra in the resulting dose distribution.

It is important to note here that these effects would also be observed with electrons transporting above the phantom surface, if they were subject to the same strong inline fields. In our experimental setup, as described in Section 2.1, there is no impact from the very low fringe field of the magnet system.

While electrons are considered dosimetrically beneficial compared to photons due to their finite range, the wide penumbra of electron beams is a limiting property to spare normal tissue close to a target volume. Earlier studies have reported the reduction of electron dose penumbra on a simulation level with conceptual magnet designs and simplistic, mono-energetic electron beams [28,29]. This work

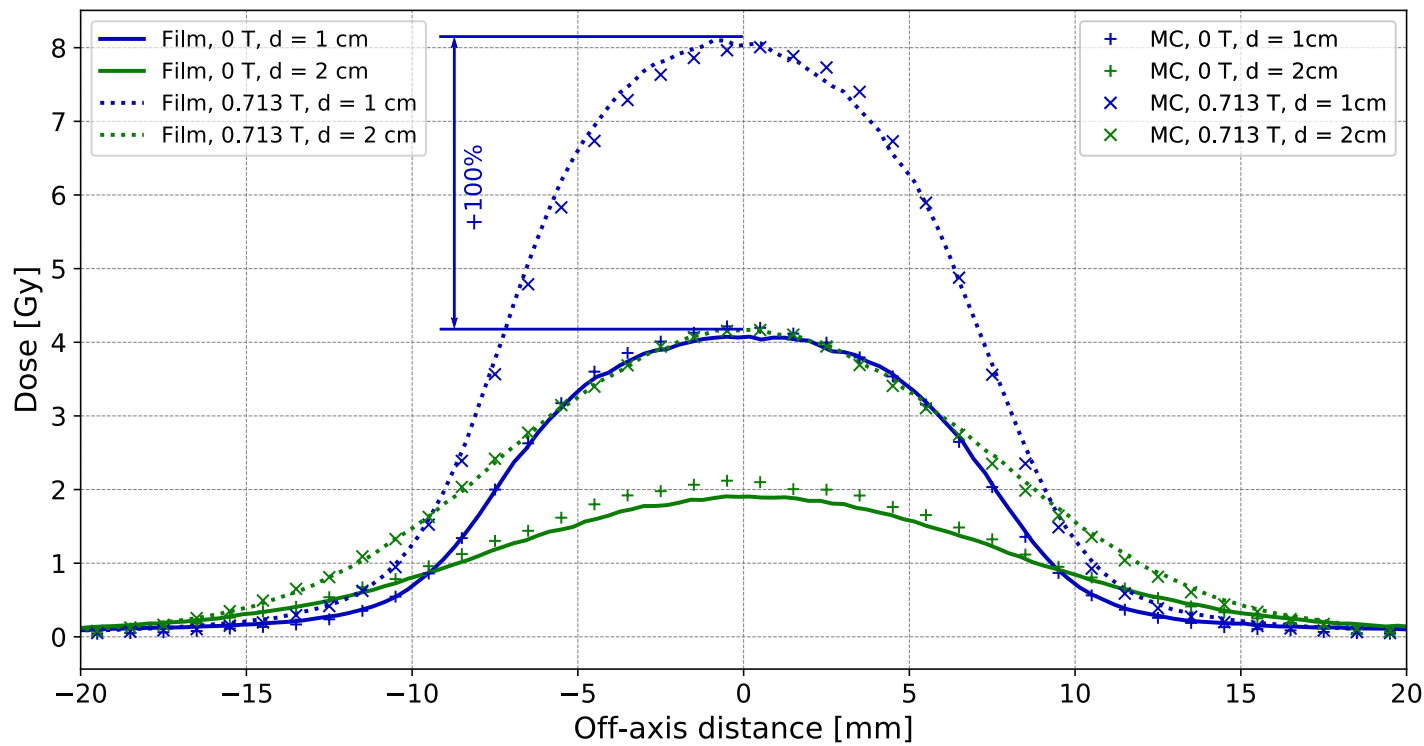

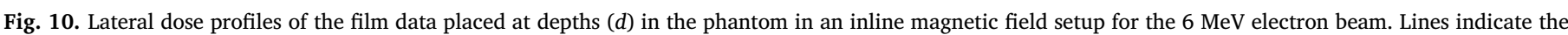

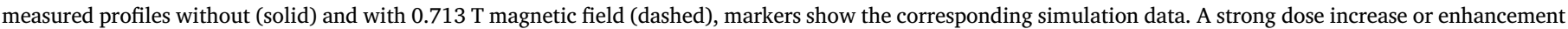
of $100 \%$ is observed at both depths. 

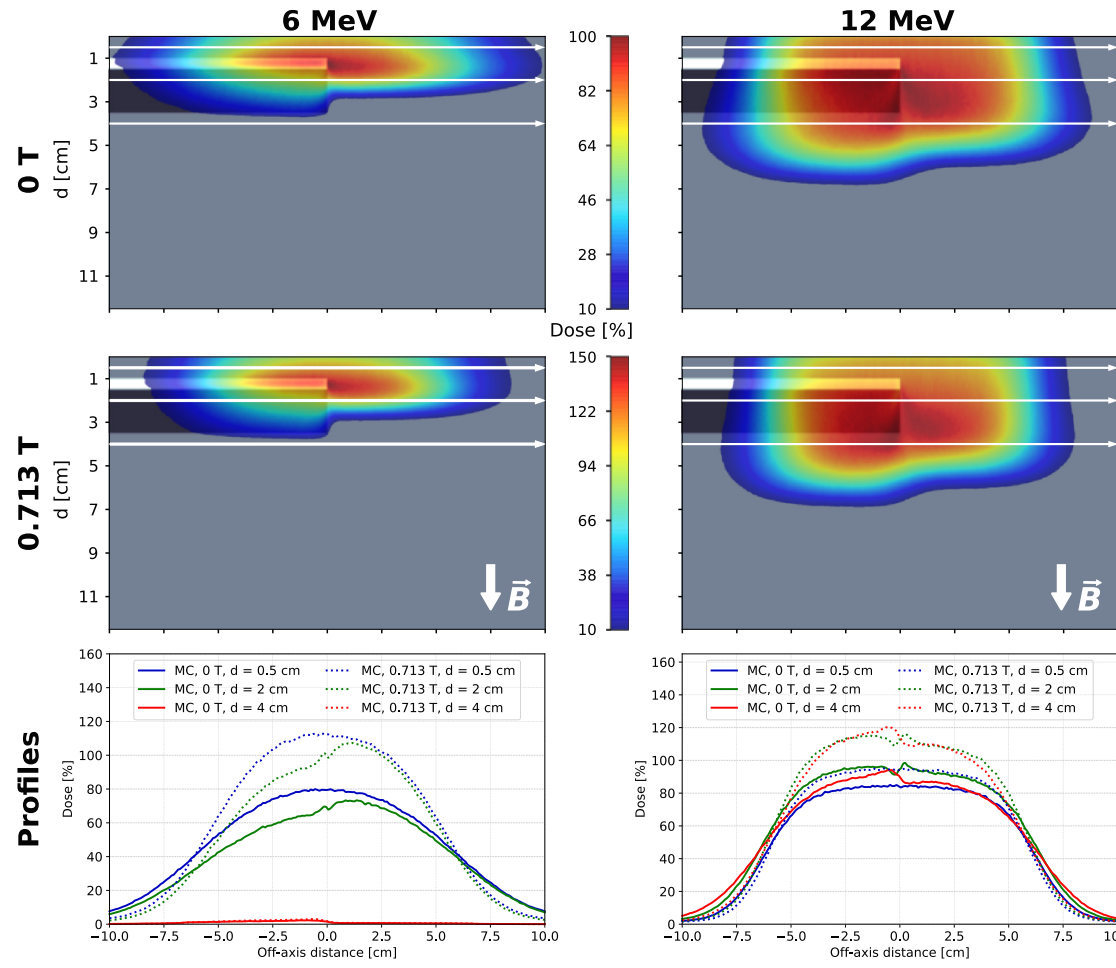
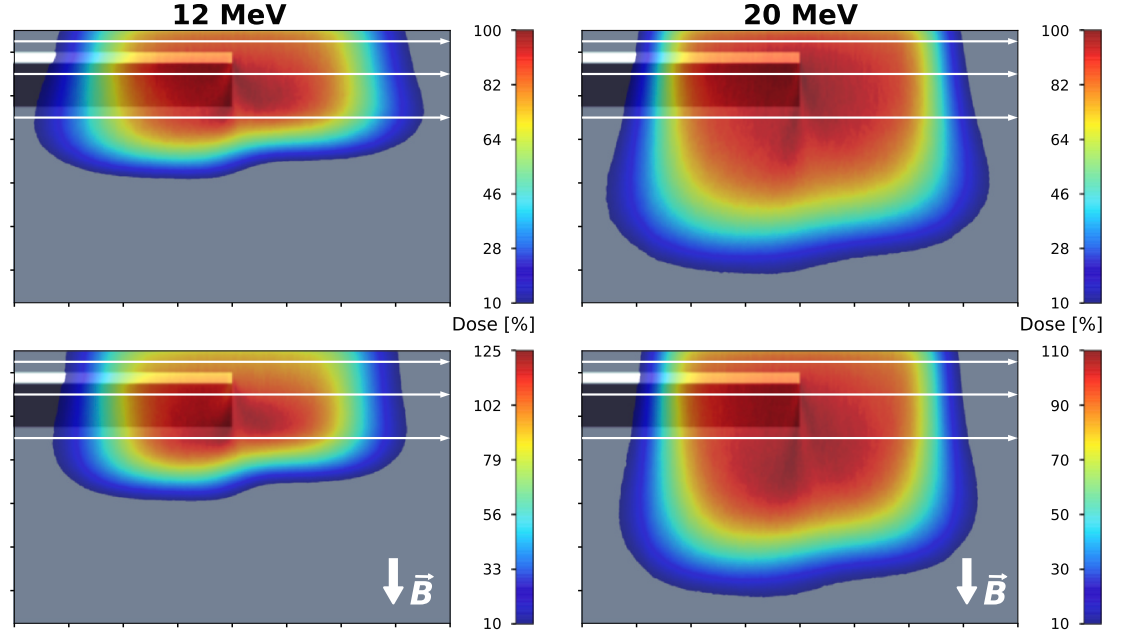

125
102
79
56
33
10

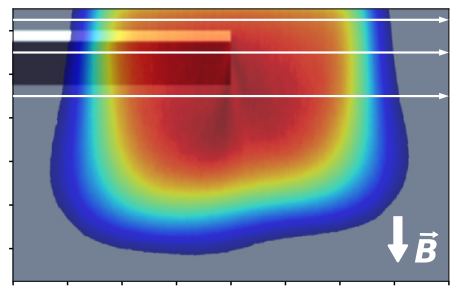
Dose $[\%]$

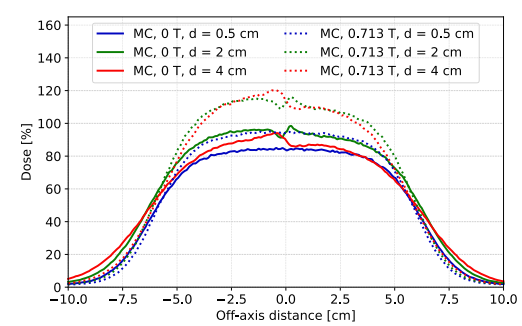

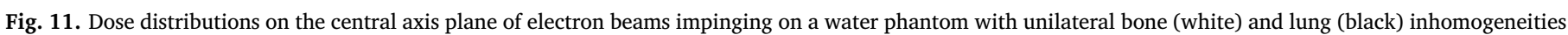

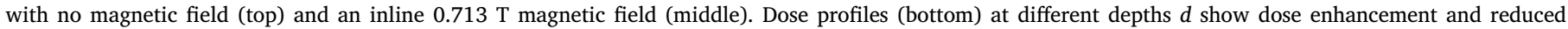
penumbral fall-off.

experimentally confirms the presumption of ameliorated dose distributions of therapeutic electron beams in the presence of local inline magnetic field similar to a MRI-linac system. Consequently, these results could be a driver towards the development of a next generation of an inline MRI-linac with an electron mode included.

\subsection{Possibility of electron beam mode in MRI-linacs}

The currently commercially available MRI-linacs are designed with the main magnetic field perpendicular to the incident photon beam $[10,11]$, which renders the possibility of an electron mode improbable. The two other far advanced projects in contrast are operating with an inline design $[8,9]$, which makes them predestined candidates for further research on this topic. It should still be noted that the present work does not cover the practicalities of producing an electron beam in a MRI-linac system. Technical challenges to generate and propagate a therapeutic electron beam in a linear accelerator remain to be solved, as the magnetic fringe field is likely to influence the treatment head. The properties of a clinical electron beam is therefore expected to be changed with a magnetic fringe field present in the accelerator head. Proper characterization of the electron beam is hence key to enable a MC based planning framework for such a concept, which remains to be addressed in future work. Nonetheless, the established framework and presented results provide a data basis for further investigation towards MRI-guided electron therapy.

\section{Conclusion}

For the first time, experiments of therapeutic energy linac-generated electron beams in both transverse and inline magnetic field directions have been performed within a common experimental and simulation framework. The results bring additional useful information on how small electron beams behave in magnetic field orientations and strengths similar to modern day MRI-linac systems. As the Monte Carlo simulations are in agreement with the measurements, we are encouraged to conduct further modeling based investigations into potential future MRI-linacs with an electron beam mode. The most promising arrangement would be an inline MRI-linac system with a photon MLC to enable dose optimization of small electron beams that inherently display a superior dose distribution over those without magnetic fields. It would then be natural process to investigate the potential benefits of mixed beam real-time MRI-guided radiotherapy.

\section{References}

[1] Dawson LA, Sharpe MB. Image-guided radiotherapy: rationale, benefits, and limitations. Lancet Oncol 2006;7(10):848-58.

[2] Bujold A, Craig T, Jaffray D, Dawson LA. Image-guided radiotherapy: has it influenced patient outcomes? In: Seminars in radiation oncology, vol. 22. Elsevier; 2012. p. 50-61.

[3] Nabavizadeh N, Elliott DA, Chen Y, Kusano AS, Mitin T, Thomas Jr CR, Holland JM. Image guided radiation therapy (IGRT) practice patterns and IGRT's impact on workflow and treatment planning: results from a National Survey of American Society for Radiation Oncology members. Int J Radiat Oncol Biol Phys 2016:94(4):850-7.

[4] Stemkens B, Tijssen RHN, de Senneville BD, Lagendijk JJW, van den Berg CAT. Image-driven, model-based 3D abdominal motion estimation for MR-guided radiotherapy. Phys Med Biol 2016;61(14):5335.

[5] Harris W, Ren L, Cai J, Zhang Y, Chang Z, Yin F-F. A technique for generating volumetric cine-magnetic resonance imaging. Int J Radiat Oncol Biol Phys 2016;95(2):844-53.

[6] Lagendijk JJW, Raaymakers BW, den Berg CAT, Moerland MA, Philippens ME, van Vulpen M, Van den Berg CAT, Moerland MA, Philippens ME, van Vulpen M, den Berg CAT, Moerland MA, Philippens ME, van Vulpen M. MR guidance in radiotherapy. Phys Med Biol 2014;59(21):R349-69. https://doi.org/10.1088/00319155/59/21/R349. http://stacks.iop.org/0031-9155/59/i=21/ $\mathrm{a}=\mathrm{R} 349$ ? key $=$ crossref.dddf92ab810f03bfacf4402a83fd09b5.

[7] Acharya S, Fischer-Valuck BW, Kashani R, Parikh P, Yang D, Zhao T, Green O, Wooten $\mathrm{O}$, Li HH, Hu Y. Others Online magnetic resonance image guided adaptive radiation therapy: first clinical applications. Int J Radiat Oncol Biol Phys 2016;94(2):394-403.

[8] Keall PJ, Barton M, Crozier S. The Australian magnetic resonance imaging-linac program. In: Seminars in radiation oncology, vol. 24. Elsevier; 2014. p. 203-6.

[9] Fallone BG. The rotating biplanar linac-magnetic resonance imaging system. In: Seminars in radiation oncology, vol. 24. Elsevier; 2014. p. 200-2.

[10] Lagendijk JJW, Raaymakers BW, Van Vulpen M. The magnetic resonance imaging-linac system. In: Seminars in radiation oncology, vol. 24. Elsevier; 2014. p. 
207-9.

[11] Mutic S, Dempsey JF. The ViewRay system: Magnetic resonance-guided and controlled radiotherapy. In: Seminars in radiation oncology, vol. 24. Elsevier; 2014. p. 196-9.

[12] Mueller S, Fix MK, Henzen D, Frei D, Frauchiger D, Loessl K, Stampanoni MF, Manser P. Electron beam collimation with a photon MLC for standard electron treatments. Physics in Medicine \& Biology 63;2. doi:10.1088/1361-6560/aa9fb6.

[13] Klein EE, Mamalui-Hunter M, Low DA. Delivery of modulated electron beams with conventional photon multi-leaf collimators. Phys Med Biol 2008;54(2):327.

[14] Salguero FJ, Arráns R, Palma BA, Leal A. Intensity-and energy-modulated electron radiotherapy by means of an xMLC for head and neck shallow tumors. Phys Med Biol 2010;55(5):1413.

[15] Alexander A, DeBlois F, Seuntjens J. Toward automatic field selection and planning using Monte Carlo-based direct aperture optimization in modulated electron radiotherapy. Phys Med Biol 2010;55(16):4563.

[16] Henzen D, Manser P, Frei D, Volken W, Neuenschwander H, Born EJ, Vetterli D, Chatelain C, Stampanoni MF, Fix MK. Monte Carlo based beam model using a photon MLC for modulated electron radiotherapy. Medical Physics 41;2. doi:10. $1118 / 1.4861711$.

[17] Mueller S, Fix MK, Joosten A, Henzen D, Frei D, Volken W, Kueng R, Aebersold DM, Stampanoni MFM, Manser P. Simultaneous optimization of photons and electrons for mixed beam radiotherapy. Phys Med Biol 2017;62(14):5840. https://doi.org/ 10.1088/1361-6560/aa70c5. http://stacks.iop.org/0031-9155/62/i =14/a =5840.

[18] Renaud M-A, Serban M, Seuntjens J. On mixed electron-photon radiation therapy optimization using the column generation approach. Med Phys 2017;44(8):4287-98.

[19] Míguez C, Jiménez-Ortega E, Palma BA, Miras H, Ureba A, Arráns R, Carrasco-Peña F, Illescas-Vacas A, Leal A. Clinical implementation of combined modulated electron and photon beams with conventional MLC for accelerated partial breast irradiation. Radiother Oncol 2017;124(1):124-9.

[20] Bostick WH. Possible techniques in direct-electron-beam tumor therapy. Phys Rev 1950;77(4):564

[21] Nardi E, Barnea G. Electron beam therapy with transverse magnetic fields. Med Phys 1999;26(6):967-73.

[22] Lee MC, Ma C-M. Monte Carlo characterization of clinical electron beams in transverse magnetic fields. Phys Med Biol 2000;45(10):2947.

[23] Whitmire DP, Bernard DL, Peterson MD, Purdy JA. Magnetic enhancement of electron dose distribution in a phantom. Med Phys 1977;4(2):127-31. https://doi. org/10.1118/1.594309. http://doi.wiley.com/10.1118/1.594309.

[24] Whitmire DP, Bernard DL, Peterson MD. Magnetic modification of the electron-dose distribution in tissue and lung phantoms. Med Phys 1978;5(5):409-17. https://doi. org/10.1118/1.594484. http://doi.wiley.com/10.1118/1.594484.

[25] Paliwal BR, Wiley AL, Wessels BW, Choi MC. Magnetic field modification of electron-beam dose distributions in inhomogeneous media. Med Phys 1978;5(5):404-8.

[26] Nath R, Schulz RJ. Modification of electron-beam dose distributions by transverse magnetic fields. Med Phys 1978;5(3):226-30.

[27] Nardi E, Barnea G, Ma C-M. Electron beam therapy with coil-generated magnetic fields. Med Phys 2004:31(6):1494-503.

[28] Weinhous MS, Nath R, Schulz RJ. Enhancement of electron beam dose distributions by longitudinal magnetic fields: Monte Carlo simulations and magnet system optimization. Med Phys 1985;12(5):598-603.

[29] Bielajew AF. The effect of strong longitudinal magnetic fields on dose deposition from electron and photon beams. Med Phys 1993;20(4):1171-9. https://doi.org/ 10.1118/1.597149. http://doi.wiley.com/10.1118/1.597149.

[30] Bielajew AF, Rogers DWO. Variance-reduction techniques. Monte Carlo transport of electrons and photons. Springer; 1988. p. 407-19.

[31] Litzenberg DW, Fraass BA, McShan DL, O’Donnell TW, Roberts DA, Becchetti FD, Bielajew AF, Moran JM. An apparatus for applying strong longitudinal magnetic fields to clinical photon and electron beams. Phys Med Biol 2001;46(5):N105.

[32] Oborn BM, Gargett MA, Causer TJ, Alnaghy SJ, Hardcastle N, Metcalfe PE, Keall PJ. Experimental verification of dose enhancement effects in a lung phantom from inline magnetic fields. Radiother Oncol 2017;125(3):433-8. https://doi.org/10. 1016/j.radonc. 2017.09.012

[33] Devic S, Seuntjens J, Sham E, Podgorsak EB, Schmidtlein CR, Kirov AS, Soares CG. Precise radiochromic film dosimetry using a flat-bed document scanner. Med Phys 2005;32(7Part1):2245-53.

[34] Fix MK, Manser P, Frei D, Volken W, Mini R, Born EJ. An efficient framework for photon Monte Carlo treatment planning. Phys Med Biol 2007;52(19):N425.

[35] Terribilini D, Fix MK, Frei D, Volken W, Manser P. VMC + + validation for photon beams in the energy range of 20-1000 keV. Med Phys 2010;37(10):5218-27.

[36] Agostinelli S, Allison J, Amako K, Apostolakis J, Araujo H, Arce P, Asai M, Axen D, Banerjee S, Barrand G, Behner F, Bellagamba L, Boudreau J, Broglia L, Brunengo A, Burkhardt H, Chauvie S, Chuma J, Chytracek R, Cooperman G, Cosmo G, Degtyarenko P, Dell'Acqua A, Depaola G, Dietrich D, Enami R, Feliciello A, Ferguson C, Fesefeldt H, Folger G, Foppiano F, Forti A, Garelli S, Giani S, Giannitrapani R, Gibin D, Cadenas JJG, González I, Abril GG, Greeniaus G, Greine W, Grichine V, Grossheim A, Guatelli S, Gumplinger P, Hamatsu R, Hashimoto K, Hasui H, Heikkinen A, Howard A, Ivanchenko V, Johnson A, Jones FW, Kallenbach J, Kanaya N, Kawabata M, Kawabata Y, Kawaguti M, Kelner S, Kent P, Kimura A, Kodama T, Kokoulin R, Kossov M, Kurashige H, Lamanna E, Lampén T, Lara V, Lefebure V, Lei F, Liendl M, Lockman W, Longo F, Magni S, Maire M, Medernach E, Minamimoto K, de Freitas PM, Morita Y, Murakami K, Nagamatu M, Nartallo R, Nieminen P, Nishimura T, Ohtsubo K, Okamura M, O'Neale S, Oohata Y, Paech K, Perl J, Pfeiffer A, Pia MG, Ranjard F, Rybin A, Sadilov S, Salvo ED, Santin G, Sasak T, Savvas N, Sawada Y, Scherer S, Sei S, Sirotenko V, Smith D, Starkov N, Stoecke H, Sulkimo J, Takahata M, Tanaka S, Tcherniaev E, Tehrani ES, Tropeano M, Truscott P, Uno H, Urban L, Urban P, Verderi M, Walkden A, Wander W, Weber H, Wellisch JP, Wenaus T, Williams DC, Wright D, Yamada T, Yoshida H, Zschiesche D. GEANT4 - A simulation toolkit. Nucl Instrum Methods Phys Res Sect A 2003;506(3):250-303.

[37] Low DA, Harms WB, Mutic S, Purdy JA. A technique for the quantitative evaluation of dose distributions. Med Phys 1998;25(5):656-61. https://doi.org/10.1118/1. 598248.

[38] Dutreix J, Dutreix A. Film dosimetry of high-energy electrons. Ann N Y Acad Sci 1969;161(1):33-43.

[39] Chen Y, Bielajew AF, Litzenberg DW, Moran JM, Becchetti FD. Magnetic confinement of electron and photon radiotherapy dose: A Monte Carlo simulation with a nonuniform longitudinal magnetic field. Med Phys 2005;32(12):3810-8.

[40] Bova FJ. A film phantom for routine film dosimetry in the clinical environment Med Dosim 1990;15(2):83-5.

[41] Khan FM, Doppke KP, Hogstrom KR, Kutcher GJ, Nath R, Prasad SC et al. Clinical electron-beam dosimetry: report of AAPM radiation therapy committee task group No. 25. Med Phys 1991;18(1):73-109. 